\title{
Robust high-order repetitive control of an active filter using an odd-harmonic internal model
}

\author{
Germán A. Ramos*, Ramon Costa-Castelló ${ }^{\dagger}$, Josep M. Olm ${ }^{\dagger}$ and Rafel Cardoner ${ }^{\dagger}$ \\ *Department of Electrical and Electronic Engineering \\ Universidad Nacional de Colombia Bogotá DC, Colombia \\ Email: garamosf@unal.edu.co \\ †Institut d’Organització i Control de Sistemes Industrials (IOC) \\ Universitat Politècnica de Catalunya, Barcelona, Spain \\ Emails: \{ramon.costa,josep.olm,rafel.cardoner\}@upc.edu
}

\begin{abstract}
Shunt active power filters have proven to be an efficient means to compensate for the negative effects of nonlinear and reactive loads on the power quality of the electrical distribution network. In this context, the control objective is to achieve a power factor close to 1 , as well as load current harmonics and reactive power compensation. A useful control strategy for this purpose is repetitive control. However, the performance of repetitive controllers is strongly affected by frequency variations of the involved signals. This work analyzes the effect of such variations and describes the architecture of an odd-harmonic, high-order repetitive controller specifically designed to obtain robust closed-loop performance against frequency variations that may occur in the electrical network.
\end{abstract}

\section{INTRODUCTION}

Active Filters (AF) are power electronics devices intended to overcome the power quality problems caused by nonlinear loads. Many research efforts have been focused on the control design of these devices [1], [2], [3], [4]. Most of them are based on two hierarchical control loops, an inner one in charge of assuring the desired current and an outer one in charge of determining the required shape as well as the appropriate power balance. In this sense, an approach which has proven to be specially efficient is Repetitive Control (RC) [5], [6]. This control technique is based on the Internal Model Principle [7] which allows the design of a controller capable of rejecting or tracking periodic signals in steady state [8], [9]. However, repetitive controllers are designed assuming a predefined fixed frequency for the signals to be tracked/rejected, and even slight changes in this frequency results in a dramatic performance decay.

In order to overcome this problem several approaches have been proposed. These methodologies may be grouped into two main frameworks, namely that dealing with sampling time preservation [10], [11] and that changing it adaptively [12], [13], [14]. The former consists of two branches: improving robustness by using large memory elements [10] and introducing a fictitious sampler operating at a variable sampling rate and then using a fixed frequency internal model [11].

The controller designed in this work uses the traditional two control loops decomposition. The current controller is composed of a feedback control law in charge of assuring closed-loop stability and a very good harmonic correction performance. For this control law, an Odd-Harmonic HighOrder Repetitive Controller (OHHORC) is proposed, which combines the specific repetitive controller structure for oddharmonic signals [15] with the High-Order Repetitive Control (HORC) design proposed in [10] to improve performance robustness under uncertain or varying frequency conditions. The outer control law is based on the exact computation of the sinusoidal current network amplitude and this is combined with a feedback control law that uses an analytically tuned PI controller.

The proposed OHHORC is the main contribution of this work, yielding very good performance and robustness under network frequency variations.

\section{PROBLEM FORMULATION}

\section{A. Physical model of the boost converter}

Fig. 1 presents the system architecture. A load is connected to the power source and an $\mathrm{AF}$ is connected in parallel to guarantee unity power factor at the network side. A boost converter with the ac neutral wire connected directly to the midpoint of the dc bus is used as AF. The averaged (at the switching frequency) model of the boost converter is given by

$$
\begin{aligned}
L \frac{d i_{f}}{d t} & =-r_{L} i_{f}-v_{1} \frac{d+1}{2}-v_{2} \frac{d-1}{2}+v_{n} \\
C_{1} \frac{d v_{1}}{d t} & =-\frac{v_{1}}{r_{C, 1}}+i_{f} \frac{d+1}{2} \\
C_{2} \frac{d v_{2}}{d t} & =-\frac{v_{2}}{r_{C, 2}}+i_{f} \frac{d-1}{2},
\end{aligned}
$$

where $d$ is the duty ratio, $i_{f}$ is the inductor current and $v_{1}, v_{2}$ are the dc capacitor voltages; $v_{n}=V_{n} \sqrt{2} \sin \left(\omega_{n} t\right)$ is the voltage source, $\omega_{n}=2 \pi / T_{p} \mathrm{rad} / \mathrm{s}$ being the network frequency; $L$ is the converter inductor and $r_{L}$ is the inductor parasitic resistance; $C_{1}, C_{2}$ are the converter capacitors and $r_{C, 1}, r_{C, 2}$ are the parasitic resistances of the capacitors. The control variable, $d$, takes its values in the closed real interval $[-1,1]$ and represents the averaged value of the pulse-width modulation control signal injected to the actual system.

Due to the nature of the voltage source, the load current, in steady-state, is usually a periodic signal with only oddharmonics in its Fourier series expansion. Hence, this current 


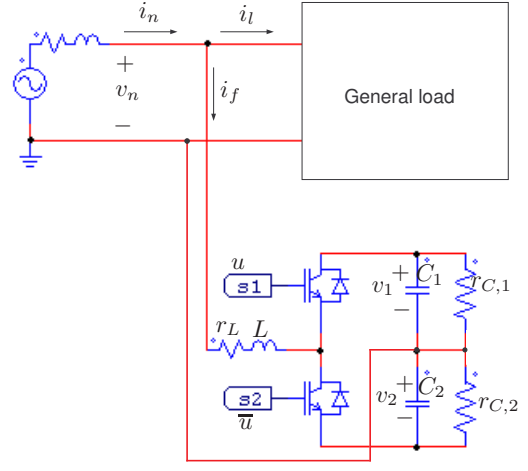

Fig. 1. Single-phase half-bridge shunt AF connected to the network-load system.

can be written as $i_{l}(t)=\sum_{n=0}^{\infty} a_{n} \sin \left(\omega_{n}(2 n+1) t\right)+$ $b_{n} \cos \left(\omega_{n}(2 n+1) t\right)$.

\section{B. Control objectives}

The AF goal is to assure that the parallel connection of AF plus load is seen as a resistive element. This can be stated demanding $i_{n}^{*}(t)=I_{d}^{*} \sin \left(\omega_{n} t\right)$, i.e. the source current must have a sinusoidal shape in phase with the network voltage ${ }^{1}$. Another collateral goal, necessary for a correct operation of the converter, is to assure constant average value of the dc bus voltage, i.e. $\left\langle v_{1}+v_{2}\right\rangle_{T_{p}}^{*}=v_{d}$, where $\langle\cdot\rangle_{T_{p}}$ stands for the mean value $^{2}$, and $v_{d}$ must fulfill the boost condition $\left(v_{d}>2 \sqrt{2} v_{n}\right)$. It is also desirable that this voltage could be almost equally distributed among both capacitors $\left(v_{1} \approx v_{2}\right)$.

\section{Rewriting the plant equations}

It is standard for this kind of systems to linearize the current dynamics by the partial state feedback $\alpha=v_{1}(d+1) / 2+$ $v_{2}(d-1) / 2$. Moreover, the change of variables $i_{f}=i_{f}$, $E_{C}=\frac{1}{2}\left(C_{1} v_{1}^{2}+C_{2} v_{2}^{2}\right), D=C_{1} v_{1}-C_{2} v_{2}$ makes two more meaningful variables appear. Namely, $E_{C}$, the energy stored in the converter capacitors and $D$, the charge unbalance between them. Assuming that the two dc bus capacitors are equal $\left(C=C_{1}=C_{2}, r_{C}=r_{C, 1}=r_{C, 2}\right)$ the system dynamics using the new variables answers to

$$
\begin{aligned}
L \frac{d i_{f}}{d t} & =-r_{L} i_{f}+v_{n}-\alpha \\
\frac{d E_{c}}{d t} & =-\frac{2 E_{c}}{r_{C} C}+i_{f} \alpha \\
\frac{d D}{d t} & =-\frac{1}{r_{C} C} D+i_{f}
\end{aligned}
$$

\section{Controller Structure}

The controller is designed using a two level approach [5], as depicted in Fig. 2. Firstly, an inner current controller which forces the sine wave shape $i_{n}^{*}$ for the network current and, second, an outer control loop to fulfill the appropriate active

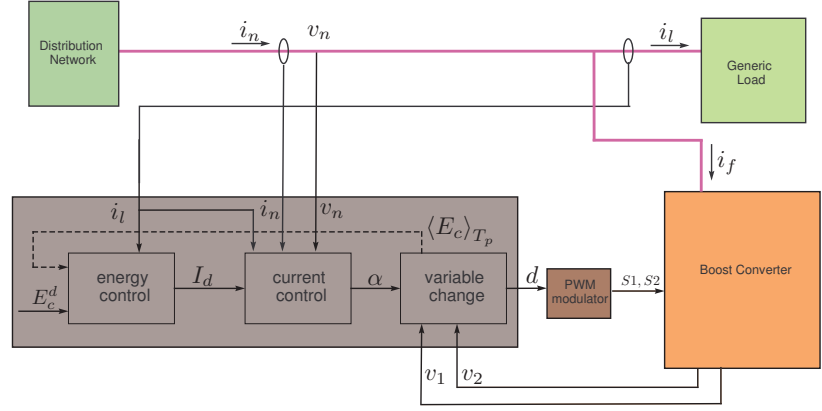

Fig. 2. Global architecture of the control system.

power balance for the whole system. The output of this loop is the amplitude of the sinusoidal reference for the current control loop. The active power balance is achieved if the energy stored in the $\mathrm{AF}$ capacitors, $E_{c}$, is equal to a reference value, $E_{c}^{d}$.

\section{A. Current loop controller}

Taking advantage of the linearity of (1), the digital repetitive controller depicted in Fig. 3 is designed to force a sinusoidal shape in $i_{n}$. The goal is to provide feedback control to overcome model uncertainties, disturbances and measurement noise.

The dynamics of (1) in discrete time can be written as

$$
G_{p}(z)=\frac{I_{f}(z)}{\alpha(z)}=\frac{1}{r_{l}} \cdot \frac{1-e^{-\frac{r_{L} T_{s}}{L}}}{z-e^{-\frac{r_{L} T_{s}}{L}}},
$$

$T_{s}$ being the sampling period. In this case, since the signal to be tracked and rejected in the system is an odd-harmonic periodic one, a technique that turns out to be specially suitable is Odd-Harmonic Repetitive Control (OHRC) [15], [16]. In this paper, an OHHORC is proposed with the aim of enhancing the performance under small frequency variations. The description of OHRC and OHHORC will be carried out in Section IV.

Under the action of the repetitive controller, the network current can be assumed to be $i_{n}(t) \approx I_{d}(t) \sin \left(\omega_{n} t\right)$ which, from now on, will be taken as a fact.

\section{B. Energy shaping (voltage loop) controller}

Following [5], the outer controller that assures a mean value of the energy stored in the capacitors, $\left\langle E_{c}(t)\right\rangle_{T_{p}}$, close to the desired reference value, $E_{c}^{d}$, is made up of two parts, as shown in Fig. 4:

(i) A feedforward term which makes $I_{d}^{f f}=a_{0}$. This assures the energy balance in the ideal case $\left(r_{L}=0\right.$ and $\left.r_{C}=0\right)$

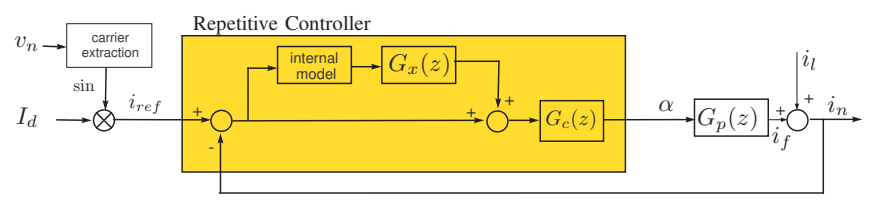

Fig. 3. Current control block diagram.
${ }^{1} f^{*}$ represents the steady-state value of $f(t)$.

${ }^{2}\langle f(t)\rangle_{T_{p}}=\frac{1}{T_{p}} \int_{t-T_{p}}^{t} f(\tau) d \tau$. 


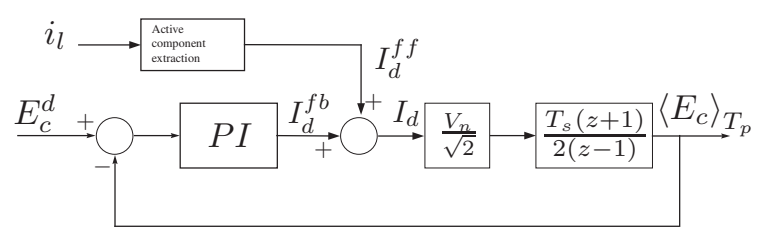

Fig. 4. Simplified $50 \mathrm{~Hz}$ energy (voltage) control loop.

and takes into account $i_{l}$ characteristics and changes instantaneously. $I_{d}^{f f}$ is calculated using an amplitude modulator with a scaled signal of the source voltage as a carrier and a mean value extraction. For this last operation, the filter

$$
P(z)=\frac{1}{N} \cdot \frac{1-z^{-N}}{1-z^{-1}}
$$

is a good approximation of the corresponding continuous-time mean value extraction.

(ii) A feedback term which is in charge of compensating dissipative effects and system uncertainties. The dynamics of the plant can be modelled by the discrete-time integrator $T_{s}(z+1) /(2(z-1))$ and the losses in the inductor and capacitors parasitic resistances can be considered as an additive disturbance. So, the PI controller

$$
I_{d}^{f b}(z)=k_{i} \frac{T_{s}(z+1)}{2(z-1)} \Delta E+k_{p} \Delta E,
$$

where $\Delta E \triangleq E_{c}^{d}-\left\langle E_{c}(t)\right\rangle_{T_{p}}$, regulates $\left\langle E_{c}(t)\right\rangle_{T_{p}}$ to the desired value $E_{c}^{d}$ without steady-state error.

\section{REPETITIVE CONTROL STRATEGIES}

\section{A. Odd-harmonic repetitive controller}

OHRC uses an internal model (Fig. 3) which introduces infinite gain at a certain frequency and its odd harmonics [15]. This internal model has the following transfer function:

$$
\frac{-H(z)}{z^{\frac{N}{2}}+H(z)},
$$

where $H(z)$ is a low-pass filter in charge of improving the system robustness. With $H(z)=1$, model (4) provides infinite gain at frequencies $\omega=2(2 k-1) \pi / N$, with $k=1,2, \ldots, N / 2+1$, where $N=T_{p} / T_{s}$ is the discrete period of the signal, $T_{p}$ being the period of the signal to be tracked/rejected and $T_{s}$ being the sampling period.

Besides the internal model, which assures steady state performance, repetitive controllers are composed of a stabilizing controller, $G_{x}(z)$, which assures closed-loop stability. Traditionally, repetitive controllers are implemented in a "plug-in" fashion, i.e. the repetitive compensator is used to augment an existing nominal controller, $G_{c}(z)$ (see Fig. 3). This nominal compensator is designed to stabilize the plant, $G_{p}(z)$, and provides disturbance attenuation across a broad frequency spectrum.

The closed-loop system of Fig. 3, using (4) as the internal model, is stable if the following conditions are fulfilled ([15]):

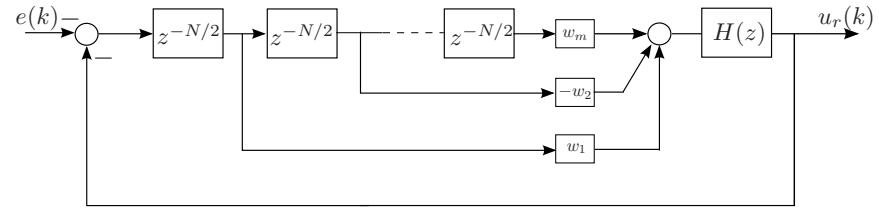

Fig. 5. OHHORC structure with an odd number of delay elements.

1) The closed-loop without the repetitive controller, i.e.

$$
G_{o}(z)=\frac{G_{c}(z) G_{p}(z)}{1+G_{c}(z) G_{p}(z)},
$$

is stable. It is advisable to design the controller $G_{c}(z)$ with a high enough robustness margin.

2) $\|H(z)\|_{\infty}<1 . H(z)$ is designed to have gain close to 1 in the desired bandwidth and attenuate the gain out of it.

3) $\left\|1-G_{o}(z) G_{x}(z)\right\|_{\infty}<1$, where $G_{x}(z)$ is a design filter to be chosen. A trivial structure ${ }^{3}$ which is often used is ([17]): $G_{x}(z)=k_{r}\left(G_{o}(z)\right)^{-1}$. As argued in [18], $k_{r}$ must be designed looking for a trade-off between robustness and transient response.

\section{B. Odd-harmonic high order repetitive controller}

HORC is mainly used to improve the RC performance robustness under disturbance/reference signals with varying or uncertain frequency [19]. Unlike standard RC, the HORC involves a weighted sum of several signal periods. With a proper selection of the associated weights, this high order function offers a characteristic frequency response in which the high gain peaks located at harmonic frequencies are extended to a wider region around the harmonics [10]. Thus, the addition of the high order function will improve robustness against frequency variations. Furthermore, the use of an odd-harmonic internal model will make the system more appropriate for applications where signals have only odd-harmonic components, as in power electronics systems.

The scheme for OHHORC is depicted in Fig. 5, its transfer function being

$$
G_{H O}(z)=\frac{-W(z) H(z)}{1+W(z) H(z)}
$$

with

$$
W(z)=\sum_{l=1}^{m}(-1)^{l-1} w_{l} z^{-\frac{l N}{2}},
$$

where $m$ is the number of delay elements in the system, and $H(z)$ is again a low-pass filter added to improve robustness.

Stability conditions for the closed-loop system of Fig. 3 using (5) as the internal model are derived in the same way as conditions given in section IV-A. Thus, conditions 1, 2 hold and an analogous version of condition 3 needs to be fulfilled:

$$
\left\|W(z) H(z)\left[1-G_{o}(z) G_{x}(z)\right]\right\|_{\infty}<1 .
$$

\footnotetext{
${ }^{3}$ There is no problem with the improperness of $G_{x}(z)$ because the internal
} model provides the repetitive controller with a high positive relative degree. 
1) Weights selection methods: The selection of the weights $w_{l}$ of the high order function $W(z)$ can give different performance characteristics to the HORC. This has led to different approaches which are primarily based on the solution of an optimization problem [20], [10], [21], [22]. The procedure described here use the maximally flat concept to calculate the weights of the function $W(z)$ in order to improve the performance robustness of the system.

Consider the internal model (5) with $H(z)=1$, namely

$$
\hat{G}_{H O}(z)=\frac{-W(z)}{1+W(z)},
$$

It can be seen that the transfer function (8) provides infinite gain when $W(z)=-1$. In the frequency domain that means

$$
W\left(e^{j \omega}\right)=\sum_{l=1}^{m}(-1)^{l-1} w_{l} e^{-\frac{j \omega l N}{2}}=-1
$$

Since it is desirable to obtain the infinite gain at odd-harmonic frequencies, it is required to set $\omega=2(2 k-1) \pi / N$ with $k=1,2,3, \ldots$ in (9), which yields the following condition

$$
\sum_{l=1}^{m} w_{l}=1
$$

This condition allows the achievement of perfect asymptotic tracking or disturbance rejection and guarantees that if the external signal is $\mathrm{N}$-periodic with odd-harmonic content, the resulting weighted sum in (6) is the same as that obtained using just one delay element.

Furthermore, it can be noticed that making $W\left(e^{j \omega}\right)$ maximally flat at odd-harmonic frequencies increases the frequency interval for which the function $W\left(e^{j \omega}\right)$ approaches -1 and, therefore, increases the interval for which the internal model (8) provides the desired high gain. As a result, the weights $w_{l}$ can be calculated using (10) and making the first $m-1$ derivatives of $W\left(e^{j \omega}\right)$ equal to 0 at odd-harmonic frequencies.

Thus, the first derivative is

$$
\frac{\partial W\left(e^{j \omega}\right)}{\partial \omega}=-j \frac{N}{2} \sum_{l=1}^{m}(-1)^{l} w_{l} l e^{-\frac{j \omega l N}{2}} .
$$

The condition states

$$
\left.\frac{\partial W\left(e^{j \omega}\right)}{\partial \omega}\right|_{\omega=\frac{2(2 k-1) \pi}{N}}=0
$$

which gives $\sum_{l=1}^{m} w_{l} l=0$. Using the same procedure to calculate the $m-1$ derivatives, the following compact condition is obtained:

$$
\sum_{l=1}^{m} w_{l} l^{p}=0, \quad p=1,2, \ldots, m-1 .
$$

Thus, for $m=3$, (10) and (11) yield $w_{1}+w_{2}+w_{3}=1$, $w_{1}+2 w_{2}+3 w_{3}=0, w_{1}+4 w_{2}+9 w_{3}=0$, which renders $w_{1}=3, w_{2}=-3$, and $w_{3}=1$.

The procedure described here attains the same conditions as those found in [10]. Also, the weights derived for HORC in [20], [21] and [22] can be used directly for OHHORC using definition (6). At the same time, the properties obtained from each method are preserved.

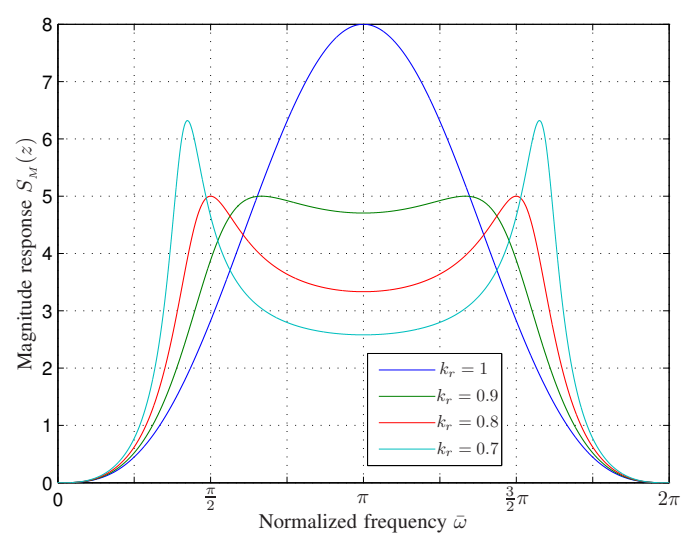

Fig. 6. $S_{M}(z)$ magnitude response for several values of $k_{r}$.

2) Selection of the gain $k_{r}$ : The sensitivity function for the OHHORC system is $S(z)=S_{o}(z) S_{M}(z)$, with

$$
S_{o}(z)=\frac{1}{1+G_{c}(z) G_{p}(z)}
$$

and $S_{M}(z)$ being the modifying sensitivity function:

$$
S_{M}(z)=\frac{1+W(z) H(z)}{1+W(z) H(z)\left[1-G_{x}(z) G_{o}(z)\right]} .
$$

The transfer function $S_{M}\left(e^{j \omega}\right)$ is periodic in the frequency domain with period $4 \pi / N$ under the assumption that $H(z)=$ 1 and $G_{x}(z)=k_{r} G_{o}^{-1}(z)$. Thus, the magnitude response between two harmonics can be described from $S_{M}\left(e^{j \omega}\right)$ using the normalized frequency $\bar{\omega}=\omega N / 2$ with $\bar{\omega} \in[\pi, 3 \pi]$ :

$$
\left|\left[S_{M}\left(e^{2 j \bar{\omega} / N}\right)\right]_{H(z)=1}\right|=\left|\frac{1+W\left(e^{2 j \bar{\omega} / N}\right)}{1+\left(1-k_{r}\right) W\left(e^{2 j \bar{\omega} / N}\right)}\right|
$$

Fig. 6 shows the magnitude of $S_{M}$ for $m=3$, with $w_{1}=3$, $w_{2}=-3, w_{3}=1$ and for several values of $k_{r}$. It can be seen that some values of the gain $k_{r}$ can be used to alleviate the over-amplification of frequencies between odd-harmonics.

\section{Performance under varying frequency conditions}

Standard RC, including the odd-harmonic version, is designed assuming the period $T_{p}$ constant. Therefore, if $T_{p}$ varies the control algorithm performance may dramatically decay. In this case, the electrical distribution network frequency can suffer from fluctuations.

As an example, Fig. 7 highlights the gain of the internal models (4) and (5), designed for a nominal frequency of $50 \mathrm{~Hz}$, for $49 \mathrm{~Hz}, 50 \mathrm{~Hz}$ and $51 \mathrm{~Hz}$ (and some of their harmonics). The selected filter is $H(z)=0.25 z+0.5+0.25 z^{-1}$. First, the magnitude of the odd-harmonic function (4) is depicted in blue. Note that while for the $50 \mathrm{~Hz}$ signal the gain is important, it strongly decays for the other frequencies. On the other hand, the magnitude of the OHHORC (5) is shown in green. It can be seen that the gain is higher for a wider frequency region around the harmonics, which improve the robustness for variations in the period $T_{p}$. As a consequence, the gain 


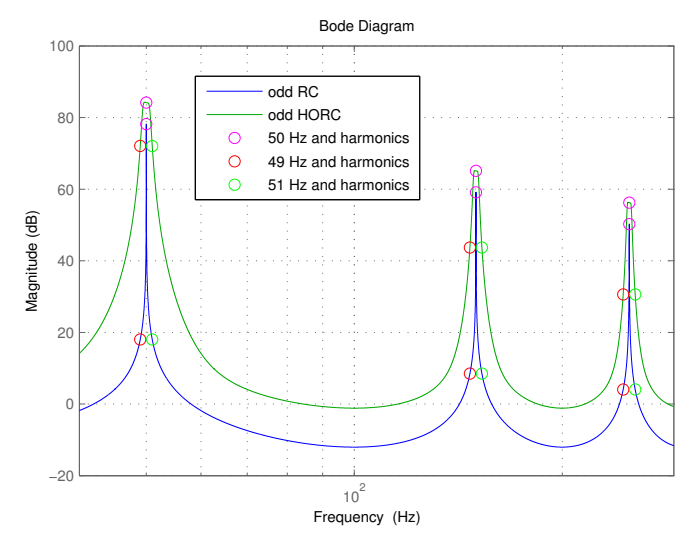

Fig. 7. OHRC and OHHORC internal models gain diagram.

decrease is much smaller for frequency variations around the nominal frequency in case of OHHORC and the performance degradation is minor.

\section{Simulations Results}

\section{A. Simulation setup}

In this section the controller scheme and the repetitive controllers described in previous sections are used in the numerical simulation of the single-phase AF defined in Section II. The controller is designed for a nominal sampling frequency of $20 \mathrm{kHz}$. The dynamics of (1) is combined with a first order low-pass anti-aliasing filter with cut-off frequency of $4.460 \mathrm{~Hz}$ and a pure delay that would occur during the real implementation. Therefore, once transformed to discrete-time the plant can be written as

$$
G_{p}(z)=\frac{I_{f}(z)}{\alpha(z)}=\frac{-0.02868 z-0.01798}{z^{3}-1.228 z^{2}+0.2417 z}
$$

The controller is designed from (12), for a nominal frequency of $50 \mathrm{~Hz}$ and obtaining 400 samples per period, i.e. $N=400$. These conditions imply a nominal sampling period of $T_{s}=T_{p} N^{-1}=50 \mu \mathrm{s}$. According to Section IV, the following design issues have been taken into account:

- $G_{c}(z)=5(0.6305 z-0.629) /(z-0.9985)$ provides a very robust inner loop.

- The first order linear-phase FIR filter $H(z)=0.25 z+$ $0.5+0.25 z^{-1}$ provides good performance in this case.

- The fact that $G_{p}(z)$ is minimum-phase allows $G_{x}(z)=$ $k_{r} G_{0}^{-1}(z)$, with $k_{r}=0.3$ for OHRC and $k_{r}=0.8$ for OHHORC.

- The weights of the high order function $W(z)$ are those derived using the procedure described in Section IV-B1 for $m=3$, which rendered $w_{1}=3, w_{2}=-3$, and $w_{3}=1$.

These settings also guarantee the fulfillment of the stability condition (7).

\section{B. Simulation results}

Fig. 8 shows the simulated waveform of $i_{n}$ and its harmonic content, which is similar to the one obtained in the real system
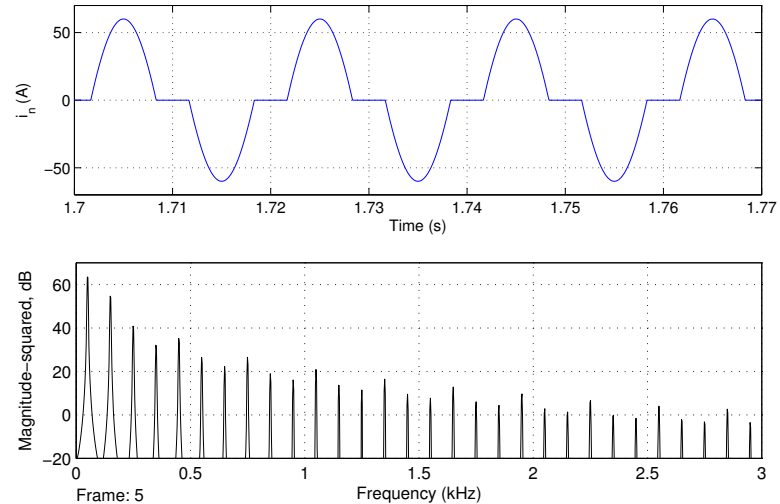

Fig. 8. Simulated $i_{n}$ waveform (top) and its harmonic content (bottom).

when a nonlinear load is connected to an ac source. The rectifier current has a Total Harmonic Distortion (THD) of $36.28 \%$.

Fig. 9 shows the shape of the current at the source port when the $\mathrm{AF}$ is connected in parallel with the rectifier. The OHRC and OHHORC controllers are compared for $50 \mathrm{~Hz}, 50.5 \mathrm{~Hz}$, and $51 \mathrm{~Hz}$. For the nominal frequency, $50 \mathrm{~Hz}$, both controllers achieve the control objectives. When the frequency is $50.5 \mathrm{~Hz}$, there is an important current shape degradation for the OHRC, while the OHHORC preserves the performance. Finally, when the frequency is set to $51 \mathrm{~Hz}$, the performance decrease is even larger for OHRC.

In Fig. 10, the harmonic content of $i_{n}$ at the nominal frequency is shown for both controllers: notice the slight improvement when using the OHHORC. The power spectrum of $i_{n}$ at $50.5 \mathrm{~Hz}$ is shown in Fig. 11; the harmonic content reveals a noticeable better performance of the OHHORC. It is also important to observe that some amplification of the frequencies placed between odd-harmonics appears in the
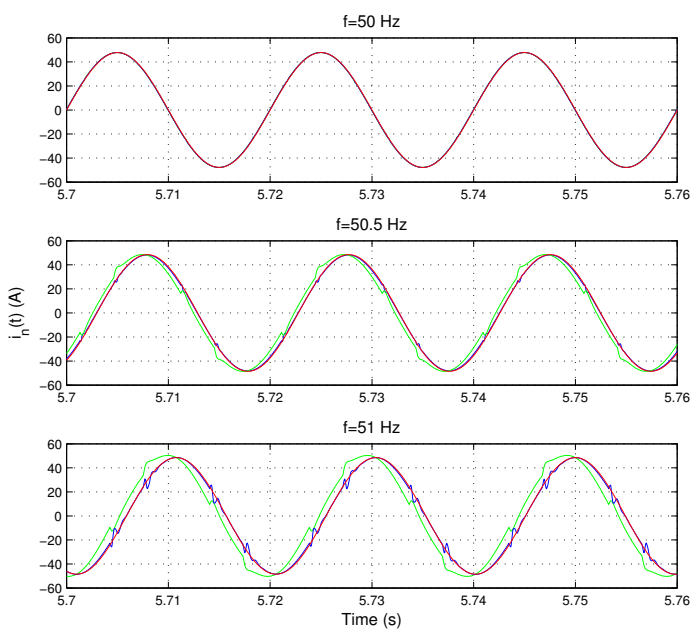

Fig. 9. Nonlinear load and the $\mathrm{AF}$ at a network frequency of $50 \mathrm{~Hz}, 50.5$ $\mathrm{Hz}$, and $51 \mathrm{~Hz} . i_{n}$ waveform for OHRC (green) and OHHORC (blue); $i_{n}^{*}$ desired waveform (red) 

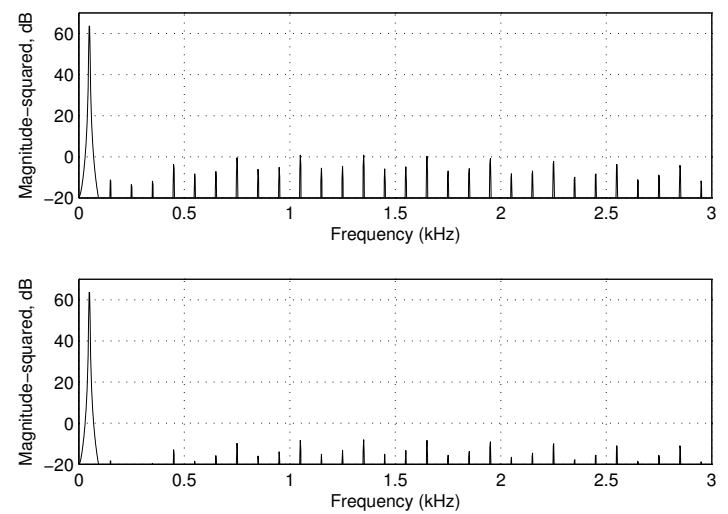

Fig. 10. Nonlinear load and the AF. Current harmonic content at $50 \mathrm{~Hz}$ OHRC (top); OHHORC (bottom).

power spectrum. This due to the fact that the HORC extends the frequency region around the odd-harmonics where the attenuation is achieved by compromising other regions of the frequency spectrum, which is known as the waterbed effect. However, given the odd harmonic characteristic of the load current signal, assuming a negligible noise level and with a proper design of the filter $\mathrm{H}(\mathrm{z})$, the performance can be preserved despite this effect.

\section{CONCLUSION}

This paper propounds an active filter controller structure based on repetitive control. As the main contribution, an oddharmonic high order repetitive controller is designed to force a sine wave shape in the current loop and to reject the harmonic content present in the load current. It has been shown that this controller provides robust performance in case of signals with uncertain or varying frequency. A comparison with an odd-harmonic repetitive controller reveals a better efficiency of the proposed controller working under varying frequency conditions.

\section{ACKNOWLEDGMENT}

This work was supported in part by the Spanish Ministerio de Educación y Ciencia under project DPI2007-62582. J.M. Olm is also supported by the Ministerio de Ciencia e Innovación through the Programa Nacional de Movilidad de Recursos Humanos of the Plan Nacional de I-D+i 2008-2011.

\section{REFERENCES}

[1] S. Choi, "A three-phase unity-power-factor diode rectifier with active input current shaping," IEEE Trans. Industrial Electronics, vol. 52, pp. 1711-1714, 2005

[2] S. Buso, L. Malesani, and P. Mattavelli, "Comparison of current control techniques for active filters applications," IEEE Trans. Industrial Electronics, vol. 45, pp. 722-729, 1998.

[3] P. Mattavelli, "A closed-loop selective harmonic compensation for active filters," IEEE Trans. Industry Applications, vol. 37, pp. 81-89, 2001.

[4] B. Singh, K. Al-Haddad, and A. Chandra, "A new control approach to Three-Phase active filter for harmonics and reactive power compensation," IEEE Trans. Power Systems, vol. 13, pp. 133-137, 1998.

[5] R. Costa-Castelló, R. Griñó, R. Cardoner-Parpal, and E. Fossas, "Highperformance control of a single-phase shunt active filter," IEEE Trans. Control Systems Technology, vol. 17, pp. 1318-1329, 2009.
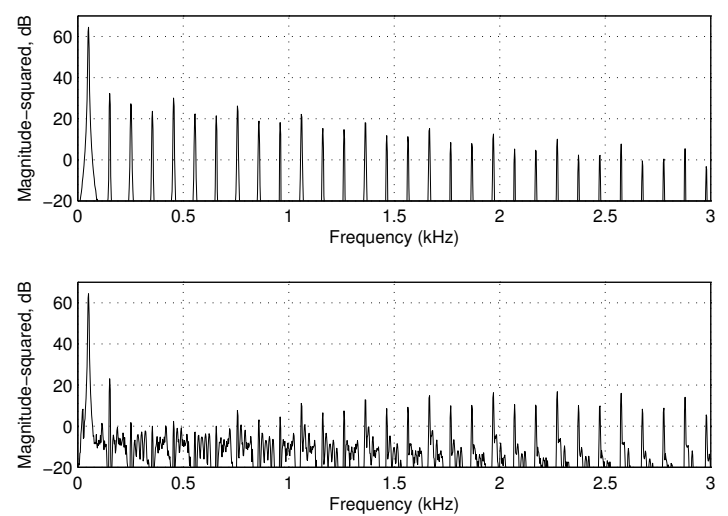

Fig. 11. Nonlinear load and the AF. Current harmonic content at $50.5 \mathrm{~Hz}$ : OHRC (top); OHHORC (bottom).

6] P. Martínez-Rodríguez, G. Escobar, M. Hernández-Gómez, and R. Torres-Olguín, "Power factor correction with an active filter using a repetitive controller," in Proc. IEEE Int. Symp. Industrial Electronics, vol. 2, 2006, pp. 1394-1399.

[7] B. Francis and W. Wonham, "Internal model principle in control theory," Automatica, vol. 12, pp. 457-465, 1976.

[8] R. Costa-Castelló, R. Griñó, and E. Fossas, "Odd-harmonic digital repetitive control of a single-phase current active filter," IEEE Trans. Power Electronics, vol. 19, pp. 1060- 1068, 2004.

[9] R. Costa-Castelló, J. Nebot, and R. Griñó, "Demonstration of the internal model principle by digital repetitive control of an educational laboratory plant," IEEE Trans. Education, vol. 48, pp. 73-80, 2005.

[10] M. Steinbuch, "Repetitive control for systems with uncertain periodtime," Automatica, vol. 38, pp. 2103-2109, 2002.

[11] Z. Cao and G. F. Ledwich, "Adaptive repetitive control to track variable periodic signals with fixed sampling rate," IEEE/ASME Trans. Mechatronics, vol. 7, pp. 374-384, 2002.

[12] G. Hillerström, "On repetitive control," Ph.D. dissertation, Lulea University of Technology, 1994.

[13] T.-C. Tsao, Y.-X. Qian, and M. Nemani, "Repetitive control for asymptotic tracking of periodic signals with an unknown period," Journal of Dynamic Systems, Measurement, and Control, vol. 122, pp. 364-369, 2000.

[14] R. D. Hanson and T.-C. Tsao, "Periodic sampling interval repetitive control and its application to variable spindle speed noncircular turning process," Journal of Dynamic Systems, Measurement, and Control, vol. 122, pp. 560-566, 2000.

[15] R. Griñó and R. Costa-Castelló, "Digital repetitive plug-in controller for odd-harmonic periodic references and disturbances," Automatica, vol. 19, pp. 1060- 1068, 2004.

[16] G. Escobar, P. Martínez, J. Leyva-Ramos, and P. Mattavelli, "A negative feedback repetitive control scheme for harmonic compensation," IEEE Trans. Industrial Electronics, vol. 53, p. 13831385, 2006.

[17] M. Tomizuka, T. Tsao, and K. Chew, "Analysis and synthesis of discretetime repetitive controllers," Journal of Dynamic Systems, Measurement, and Control, vol. 111, pp. 353-358, September 1989.

[18] G. Hillerström and R. C. Lee, "Trade-offs in repetitive control," University of Cambridge, Tech. Rep. CUED/F-INFENG/TR 294, June 1997.

[19] T. Inoue, "Practical repetitive control system design," in Proc. 29th IEEE Conference on Decision and Control, 1990, pp. 1673-1678.

[20] W. S. Chang, I. H. Suh, and T. W. Kim, "Analysis and design of two types of digital repetitive control systems," Automatica, vol. 31, pp. 741-746, 1995.

[21] M. Steinbuch, S. Weiland, and T. Singh, "Design of noise and periodtime robust high order repetitive control, with application to optical storage," Automatica, vol. 43, pp. 2086-2095, 2007.

[22] G. Pipeleers, B. Demeulenaere, and S. Sewers, "Robust high order repetitive control: Optimal performance trade offs," Automatica, vol. 44, pp. 2628-2634, 2008. 